\title{
On a functional equation related to competition
}

\author{
Peter Kahlig and Janusz Matkowski
}

Abstract. The functional equation

$$
f\left(\frac{x+y}{1-x y}\right)=\frac{f(x)+f(y)}{1+f(x) f(y)}, \quad x y<1,
$$

(introduced by the first author in a competition model) is considered. The main result says that a function $f: \mathbb{R} \rightarrow \mathbb{R}$ satisfies this equation if, and only if, $f=\tanh \circ \alpha \circ \tan ^{-1}$, where $\alpha: \mathbb{R} \rightarrow \mathbb{R}$ is an additive function.

Mathematics Subject Classification (1991). Primary 39B12, 39 B22.

Keywords. Functional equation, additive function, general solution, competition model.

\section{Introduction}

Motivated by a model of competition coming from cloud physics, the firstnamed author $[1,2]$ introduced the following functional equation

$$
f\left(\frac{x+y}{1-x y}\right)=\frac{f(x)+f(y)}{1+f(x) f(y)}, \quad(x, y) \in \mathbb{R}^{2}, \quad x y \neq 1 .
$$

Applying a uniqueness result [3] for a related equation in a single variable, the form of solutions under some special regularity conditions was established (cf. Remark 5).

In Sect. 2 we present properties of solutions of this equation which in a natural way lead to the consideration of Eq. (1) with the domain restricted to the set $\left\{(x, y) \in \mathbb{R}^{2}: x y<1\right\}$. We prove, among other things, that if $f: \mathbb{R} \rightarrow \mathbb{R}$ satisfies this equation and $f\left(y_{0}\right)=1$ or $f\left(y_{0}\right)=-1$ for some $y_{0} \in \mathbb{R}$, then $f$ is a constant function (Proposition 1). Moreover $f(0)$ is either 0 or -1 or 1 . If $f(0)=0$ then $f$ is an odd function. In Sect. 3 we prove that the function $f=\tanh \circ \alpha \circ \tan ^{-1}$, where $\alpha: \mathbb{R} \rightarrow \mathbb{R}$ is an arbitrary additive function, is the general solution. As a corollary we obtain that, under some weak regularity 
conditions, every solution must be of the form $f(x)=\tanh \left(c \tan ^{-1}(x)\right)(x \in$ $\mathbb{R})$ for some $c \in \mathbb{R}$.

\section{Properties of solutions of the functional equation}

Since the function $(x, y) \rightarrow \frac{x+y}{1-x y}$ occurring in Eq. (1) is not defined on the set $\left\{(x, y) \in \mathbb{R}^{2}: x y=1\right\}$, instead of Eq. (1), it is natural to consider the following two functional equations on a restricted domain:

$$
f\left(\frac{x+y}{1-x y}\right)=\frac{f(x)+f(y)}{1+f(x) f(y)}, \quad(x, y) \in \mathbb{R}^{2}, x y<1,
$$

and

$$
f\left(\frac{x+y}{1-x y}\right)=\frac{f(x)+f(y)}{1+f(x) f(y)}, \quad(x, y) \in \mathbb{R}^{2}, x y>1 .
$$

Remark 1 . Since $\{x \in \mathbb{R}: x y<1$ for some $y \in \mathbb{R}\}=\mathbb{R}$, it is reasonable to ask for solutions of the type $f: \mathbb{R} \rightarrow \mathbb{R}$ of Eq. (2), that are defined on the whole $\mathbb{R}$.

Note that this problem makes no sense in the case of Eq. (3), as no point $(x, y)$ with $x=0$ satisfies the condition $x y>1$. Moreover, the domain of Eq. (3), the set $D:=\{(x, y): x y>1\}$ is the sum of two disjoint open connected sets $D_{+}:=\left\{(x, y): x>0 \wedge y>\frac{1}{x}\right\}$ and $D_{-}:=\left\{(x, y): x<0 \wedge y<\frac{1}{x}\right\}$. Since $\frac{x+y}{1-x y}<0 \quad$ for all $(x, y) \in D_{+} \quad$ and $\quad \frac{x+y}{1-x y}>0 \quad$ for all $(x, y) \in D_{-}$,

neither the problem to find a solution $f:(0, \infty) \rightarrow \mathbb{R}$ nor the problem to find a solution $f:(-\infty, 0) \rightarrow \mathbb{R}$ make sense in the case of Eq. (3).

(These facts show that in the case of Eq. (3) one could look for solutions $f:[(-\infty, 0) \cup(0, \infty)] \rightarrow \mathbb{R}$.)

Note also that if $f: \mathbb{R} \rightarrow \mathbb{R}$ satisfies (1) then, clearly, it satisfies (2), and its restriction to $\mathbb{R} \backslash\{0\}$ satisfies (3).

We begin with the following:

Proposition 1. Suppose that a function $f: \mathbb{R} \rightarrow \mathbb{R}$ satisfies Eq. (1). Then

(i) the function $-f$ satisfies Eq. (1);

(ii) if $f\left(y_{0}\right)=1$ for some $y_{0} \in \mathbb{R}$ then $f(x)=1$ for all $x \in \mathbb{R}$;

(iii) if $f\left(y_{0}\right)=-1$ for some $y_{0} \in \mathbb{R}$ then $f(x)=-1$ for all $x \in \mathbb{R}$.

Proof. The result (i) is obvious.

To prove (ii) assume that $f\left(y_{0}\right)=1$ for some $y_{0} \in \mathbb{R}$. If $y_{0}=0$, setting $y=y_{0}=0$ in $(1)$, we get

$$
f(x)=\frac{f(x)+1}{1+f(x)}=1, \quad x \in \mathbb{R} .
$$


If $y_{0}>0$ we get

$$
f\left(\frac{x+y_{0}}{1-x y_{0}}\right)=\frac{f(x)+1}{1+f(x)}=1, \quad x<\frac{1}{y_{0}} .
$$

Since the range of the function

$$
\left(-\infty, \frac{1}{y_{0}}\right) \ni x \longmapsto \frac{x+y_{0}}{1-x y_{0}}
$$

is the interval $\left(-\frac{1}{y_{0}}, \infty\right)$, we get

$$
f(x)=1, \quad x \in\left(-\frac{1}{y_{0}}, \infty\right),
$$

in particular $f(x)=1$ for all $x \in(0, \infty)$. Taking $y_{0}$ arbitrarily close to 0 from the right, we obtain

$$
f(x)=1, \quad x \in(-\infty, \infty),
$$

which was to be shown. If $y_{0}<0$, the argument is similar.

We omit an analogous proof of (iii).

Proposition 2. Neither Eq. (2) nor Eq. (3) has a solution that is continuous at a point $x_{0}$ and unbounded in a vicinity of 0 .

Proof. Assume that $f$ satisfies Eq. (2) or Eq. (3), is continuous at the point $x_{0}$, and there exists a sequence $\left(y_{n}\right)$ such that $\lim _{n \rightarrow \infty} y_{n}=0$ and $\lim _{n \rightarrow \infty}\left|f\left(y_{n}\right)\right|$ $=\infty$. Of course $f\left(x_{0}\right) \neq 0$. Then

$$
\begin{aligned}
f\left(x_{0}\right) & =\lim _{n \rightarrow \infty} f\left(\frac{x_{0}+y_{n}}{1-x_{0} y_{n}}\right)=\lim _{n \rightarrow \infty} \frac{f\left(x_{0}\right)+f\left(y_{n}\right)}{1+f\left(x_{0}\right) f\left(y_{n}\right)} \\
& =\lim _{n \rightarrow \infty} \frac{\frac{f\left(x_{0}\right)}{f\left(y_{n}\right)}+1}{\frac{1}{f\left(y_{n}\right)}+f\left(x_{0}\right)}=\frac{1}{f\left(x_{0}\right)},
\end{aligned}
$$

which implies that $f\left(x_{0}\right)=1$ or $f\left(x_{0}\right)=-1$. In view of Proposition 1 the function $f$ would be constant, contradicting the assumption.

In the sequel we shall deal with the functional equation (2).

Remark 2. If $f: \mathbb{R} \rightarrow \mathbb{R}$ satisfies Eq. (2), then either $f(0)=0$ or $f(0)=1$ or $f(0)=-1$.

Indeed, setting $x=y=0$ in (2) we get

$$
f(0)\left([f(0)]^{2}-1\right)=0 .
$$

Hence, making use of Proposition 1, we obtain:

Corollary 1. If $f: \mathbb{R} \rightarrow \mathbb{R}$ satisfies Eq. (2) then either $f(0)=0$ or $f$ is a constant function of the value 1 or -1 . 
Therefore in the sequel we are mainly interested in the solutions $f: \mathbb{R} \rightarrow \mathbb{R}$ of Eq. (2) such that $f(0)=0$.

Remark 3. If $f: \mathbb{R} \rightarrow \mathbb{R}$ satisfies equation (2) and $f(0)=0$ then $f$ is an odd function.

Indeed, for $y=-x$ we have $x y=-x^{2}<1$ and, setting $y=-x$ in Eq. (2), we obtain

$$
0=f(0)=\frac{f(x)+f(-x)}{1+f(x) f(-x)},
$$

whence $f(-x)=-f(x)$ for all $x \in \mathbb{R}$.

Remark 4. Suppose that $f: \mathbb{R} \rightarrow \mathbb{R}$ satisfies Eq. (2). If $f\left(y_{0}\right)=0$ for some $y_{0} \neq 0$, then $f(0)=0$ and

$$
f\left(\frac{x+y_{0}}{1-x y_{0}}\right)=f(x), \quad x \in \mathbb{R} .
$$

Indeed, in view of Corollary 1 , we have $f(0)=0$. The remaining part of this remark one gets immediately by setting $y=y_{0}$ in (2).

From Proposition 1 and Corollary 1 we obtain the following:

Corollary 2. Suppose that $f: \mathbb{R} \rightarrow \mathbb{R}$ is a continuous solution of Eq. (2). Then the following conditions are pairwise equivalent:

(i) there exist $x_{1}, x_{2} \in \mathbb{R}$ such that $f\left(x_{1}\right) \neq 1$ and $f\left(x_{2}\right) \neq-1$;

(ii) there exists $x_{0} \in \mathbb{R}$ such that $f\left(x_{0}\right) \in(-1,1)$;

(iii) $f(0)=0$;

(iv) $|f(x)|<1$ for all $x \in \mathbb{R}$.

Remark 5. Setting $y=x$ in Eq. (2) we obtain the following functional equation in a single variable

$$
f\left(\frac{2 x}{1-x^{2}}\right)=\frac{2 f(x)}{1+[f(x)]^{2}}, \quad|x|<1,
$$

which is used in $[1,2]$.

Assume that $f: \mathbb{R} \rightarrow \mathbb{R}$ satisfies Eq. (1). Replacing $y$ in (1) by $\frac{y+z}{1-y z}$ we obtain

$f\left(\frac{x+y+z-x y z}{1-x y-x z-y z}\right)=\frac{f(x)+f(y)+f(z)+f(x y z)}{1+f(x y)+f(x z)+f(y z)}, \quad x y+x z+y z \neq 1$,

whence, setting $z=y=x$, we obtain the following functional equation in a single variable

$$
f\left(\frac{3 x-x^{3}}{1-3 x^{2}}\right)=\frac{3 f(x)+f\left(x^{3}\right)}{1+3 f\left(x^{2}\right)}, \quad 3 x^{2}<1 .
$$


By induction, this procedure and Eq. (1) lead to the following infinite system of functional equations of $n$ variables $x_{1}, \ldots, x_{n}$,

$$
f\left(\frac{\sum_{j=1}^{\left[\frac{n+1}{2}\right]}(-1)^{j} \sum_{i_{1}<\ldots<i_{2 j-1}} \prod_{k=1}^{2 j-1} x_{i_{k}}}{1-\sum_{j=1}^{\left[\frac{n+1}{2}\right]}(-1)^{j} \sum_{i_{1}<\ldots<i_{2 j}} \prod_{k=1}^{2 j} x_{i_{k}}}\right)=\frac{\sum_{j=1}^{\left[\frac{n+1}{2}\right]} \sum_{i_{1}<\ldots<i_{2 j-1}} \prod_{k=1}^{2 j-1} f\left(x_{i_{k}}\right)}{1+\sum_{j=1}^{\left[\frac{n+1}{2}\right]} \sum_{i_{1}<\ldots<i_{2 j}} \prod_{k=1}^{2 j} f\left(x_{i_{k}}\right)},
$$

where $n \in \mathbb{N}, n \geq 2$, and $x_{1}, \ldots, x_{n} \in \mathbb{R}$ are such that

$$
\sum_{j=1}^{\left[\frac{n+1}{2}\right]}(-1)^{j} \sum_{i_{1}<\ldots<i_{2 j}} \prod_{k=1}^{2 j} x_{i_{k}} \neq 1
$$

(here $\left[\frac{n+1}{2}\right]$ denotes the largest integer not greater than $\frac{n+1}{2}$ ). Setting here $x_{1}, \ldots, x_{n}=x$ we obtain for $f$ the system of iterative functional equations

$$
f\left(\frac{\sum_{j=1}^{\left[\frac{n+1}{2}\right]}(-1)^{j}\left(\begin{array}{c}
n \\
2 j-1
\end{array}\right) x^{2 j-1}}{1-\sum_{j=1}^{\left[\frac{n+1}{2}\right]}(-1)^{j}\left(\begin{array}{c}
n \\
2 j
\end{array}\right) x^{2 j}}\right)=\frac{\sum_{j=1}^{\left[\frac{n+1}{2}\right]}(-1)^{j}\left(\begin{array}{c}
n \\
2 j-1
\end{array}\right) f(x)^{2 j-1}}{1-\sum_{j=1}^{\left[\frac{n+1}{2}\right]}(-1)^{j}\left(\begin{array}{c}
n \\
2 j
\end{array}\right) f(x)^{2 j}},
$$

for all $n \in \mathbb{N}, n \geq 2$, and $x \in \mathbb{R}$ such that $\sum_{j=1}^{\left[\frac{n+1}{2}\right]}(-1)^{j}\left(\begin{array}{c}n \\ 2 j\end{array}\right) x^{2 j} \neq 1$.

\section{Main result}

Theorem 1. A function $f: \mathbb{R} \rightarrow \mathbb{R}$ satisfies the functional equation (2) if, and only if, there exists an additive function $\alpha: \mathbb{R} \rightarrow \mathbb{R}$ such that

$$
f=\tanh \circ \alpha \circ \tan ^{-1} \text {. }
$$

Proof. We have the identity

$$
\frac{x+y}{1-x y}=\tan \left(\tan ^{-1} x+\tan ^{-1} y\right), \quad x y<1 .
$$

Since

$$
\frac{\tanh x+\tanh y}{1+(\tanh x)(\tanh y)}=\tanh (x+y), \quad x, y \in \mathbb{R},
$$

we also have the identity

$$
\frac{u+v}{1+u v}=\tanh \left(\tanh ^{-1}(u)+\tanh ^{-1}(v)\right), \quad u, v \in(-1,1) .
$$


Hence, assuming that $f: \mathbb{R} \rightarrow \mathbb{R}$ satisfies Eq. (2), we obtain

$f\left(\tan \left(\tan ^{-1} x+\tan ^{-1} y\right)\right)=\tanh \left(\tanh ^{-1}(f(x))+\tanh ^{-1}(f(y))\right), \quad x y<1$.

Setting here $u=\tan ^{-1} x, v=\tan ^{-1} y$, we obtain

$$
\tanh ^{-1} \circ f \circ \tan (u+v)=\tanh ^{-1} \circ f \circ \tan (u)+\tanh ^{-1} \circ f \circ \tan (v)
$$

for all $u, v \in \mathbb{R}$ such that $(\tan u)(\tan v)<1$.

It follows that the function $\alpha:\left(-\frac{\pi}{2}, \frac{\pi}{2}\right) \rightarrow \mathbb{R}$ defined by $\alpha:=\tanh ^{-1} \circ f \circ \tan$ satisfies the Cauchy functional equation

$$
\alpha(u+v)=\alpha(u)+\alpha(v), \quad u, v \in \mathbb{R}, \quad(\tan u)(\tan v)<1 .
$$

As the $\operatorname{set}\left\{(u, v) \in \mathbb{R}^{2}:(\tan u)(\tan v)<1\right\}$ is an open connected set such that $(0,0)$ is its interior point, the function $\alpha$ has a unique additive extension defined on $\mathbb{R}$. Without any loss of generality, we can denote it also by $\alpha$. Thus we have shown that if $f: \mathbb{R} \rightarrow \mathbb{R}$ satisfies Eq. (2), then there exists an additive function $\alpha: \mathbb{R} \rightarrow \mathbb{R}$ such that

$$
f=\tanh \circ \alpha \circ \tan ^{-1} .
$$

To prove the converse implication assume that $f: \mathbb{R} \rightarrow \mathbb{R}$ is of this form. Then, making use of the additivity of $\alpha$ and the properties of the functions tanh and tan, we have for all $x, y \in \mathbb{R}$ such that $x y<1$,

$$
\begin{aligned}
f\left(\frac{x+y}{1-x y}\right) & =\tanh \circ \alpha \circ \tan ^{-1}\left(\frac{x+y}{1-x y}\right) \\
& =\tanh \left(\alpha\left[\tan ^{-1}\left(\frac{\tan \left(\tan ^{-1} x\right)+\tan \left(\tan ^{-1} y\right)}{1-\tan \left(\tan ^{-1} x\right) \cdot \tan \left(\tan ^{-1} y\right)}\right)\right]\right) \\
& =\tanh \left(\alpha\left[\tan ^{-1}\left(\tan \left(\tan ^{-1} x+\tan ^{-1} y\right)\right)\right]\right) \\
& =\tanh \left(\alpha\left[\tan ^{-1} x+\tan ^{-1} y\right]\right)=\tanh \left(\alpha\left(\tan ^{-1} x\right)+\alpha\left(\tan ^{-1} y\right)\right) \\
& =\frac{\tanh \left(\alpha\left(\tan ^{-1} x\right)\right)+\tanh \left(\alpha\left(\tan ^{-1} y\right)\right)}{1+\left(\tanh \left(\alpha\left(\tan ^{-1} x\right)\right)\right)\left(\tanh \left(\alpha\left(\tan ^{-1} y\right)\right)\right)} \\
& =\frac{\tanh \circ \circ \circ \tan ^{-1}(x)+\tanh \circ \alpha \circ \tan ^{-1}(y)}{1+\left[\tanh \circ \alpha \circ \tan ^{-1}(x)\right]\left[\tanh \circ \alpha \circ \tan ^{-1}(y)\right]}=\frac{f(x)+f(y)}{1+f(x) f(y)} .
\end{aligned}
$$

This completes the proof.

Remark 6. The family of all solutions of Eq. (2) is extremely big in the following sense: for each point $\left(x_{0}, y_{0}\right) \in \mathbb{R}^{2}$ such that $x_{0} \neq 0$ and $y_{0} \in(-1,1)$ there exists a continuum of different solutions of the form $f=\tanh \circ \alpha \circ \tan ^{-1}$ with an additive function $\alpha$ such that $f\left(x_{0}\right)=y_{0}$.

Corollary 3. Suppose that $f: \mathbb{R} \rightarrow \mathbb{R}$ satisfies one of the following conditions:

(i) $f$ is continuous at a point;

(ii) $f$ is measurable in the sense of Lebesgue; 
(iii) $f$ is bounded from above or bounded from below on a set of positive Lebesgue measure;

(iv) the graph of $f$ is not dense in $\mathbb{R}^{2}$.

Then $f$ satisfies the functional equation (2),

$$
f\left(\frac{x+y}{1-x y}\right)=\frac{f(x)+f(y)}{1+f(x) f(y)}, \quad x y<1,
$$

[or Eq. (1)] if, and only if, there exists a constant $c \in \mathbb{R}$ such that

$$
f=\tanh \circ\left(c \tan ^{-1}\right) .
$$

Proof. Since $\alpha:=\tanh ^{-1} \circ f \circ \tan$, it satisfies one of the conditions (i), (ii), (iii), (iv) and is an additive function. This implies (cf. M. Kuczma [4]) that there exists a constant $c \in \mathbb{R}$ such that $\alpha(u)=c u, \quad u \in \mathbb{R}$.

Hence, taking also into account Proposition 1, we obtain

Remark 7. The family of regular solutions of Eq. (2) [Eq. (1)] [i.e. satisfying one of conditions (i)-(iv)] form a one-parameter family of functions such that for each point $\left(x_{0}, y_{0}\right) \in \mathbb{R}^{2}$ such that $x_{0} \neq 0$ and $y_{0} \in[-1,1]$ there exists a unique solution $f: \mathbb{R} \rightarrow \mathbb{R}$ of Eq. (2) [Eq. (1)] such that $f\left(x_{0}\right)=y_{0}$. Moreover, if $y_{0} \in(-1,1)$ then

$$
f=\tanh \circ\left(\frac{\tanh ^{-1}\left(y_{0}\right)}{\tan ^{-1}\left(x_{0}\right)} \tan ^{-1}\right) ;
$$

if $y_{0}=-1$ then $f=-1$; if $y_{0}=1$ then $f=1$.

In particular the sum of all graphs of this family of solutions is the set

$$
((\mathbb{R} \backslash\{0\}) \times[-1,1]) \cup(\{0\} \times\{-1,0,1\}) .
$$

\section{Acknowledgements}

The referee's helpful comments are acknowledged with thanks.

Open Access. This article is distributed under the terms of the Creative Commons Attribution License which permits any use, distribution, and reproduction in any medium, provided the original author(s) and the source are credited.

\section{References}

[1] Kahlig, P.: A model of competition. Appl. Math. 39, 293-303 (2012)

[2] Kahlig, P.: Note to the paper "A model of competition". Appl. Math. 40, 127 (2013)

[3] Matkowski, J.: The uniqueness of solutions of a system of functional equations in some classes of functions. Aequ. Math. 8, 233-237 (1972) 
[4] Kuczma, M.: An introduction to the theory of functional equations and inequalities. Cauchy's equation and Jensen's inequality. Uniwersytet Śląski - PWN, Warszawa Krakow - Katowice, 1985 (2nd edn., edited with a preface of Attila Gilányi, Birkhäuser verlag, Basel, 2009)

Peter Kahlig

Altmannsdorfer Str., 21/5/2

1120 Vienna

Austria

e-mail: peter.kahlig@gmx.at

Janusz Matkowski

Faculty of Mathematics

Computer Science and Econometrics

University of Zielona Góra

Szafrana 4A

65-516 Zielona Góra

Poland

e-mail: J.Matkowski@wmie.uz.zgora.pl

Received: November 15, 2012

Revised: April 19, 2013 